\title{
CHEMICAL AND SENSORY CHARACTERISATION OF SWEET WINES OBTAINED BY DIFFERENT TECHNIQUES
}

\section{CARACTERIZAÇÃO QUÍMICA E SENSORIAL DE VINHOS DOCES OBTIDOS POR DIFERENTES TÉCNICAS}

\author{
José-Miguel Avizcuri-Inac ${ }^{1,3,4}$, Marivel González-Hernández ${ }^{1,4}$, Daniel Rosáenz-Oroz ${ }^{5}$, Rodrigo Martínez- \\ Ruiz $^{1}$, Luis Vaquero-Fernández ${ }^{1,2} *$ \\ ${ }^{1}$ Department of Chemistry and ${ }^{2}$ Laboratory Service. University of La Rioja. Madre de Dios, 53. 26006. Logroño, Spain. \\ ${ }^{3}$ Bodegas Ontañón S.L. Machín sn, 26559. Aldeanueva de Ebro, Spain. \\ ${ }^{4}$ Institute of Vine and Wine Sciences (UR, CSIC, GR). Finca La Grajera, Autovía del Camino de Santiago LO-20 Salida 13, 26007. Logroño, \\ Spain. \\ ${ }^{5}$ Sociedad Cooperativa de Albelda, Escuelas Pías, 20, 26120. Albelda de Iregua, Spain.
}

* Corresponding author: Tel: +34 941299699, e-mail: luis.vaquero@unirioja.es

(Received 13.01.2018. Accepted 19.03.2018)

\section{SUMMARY}

Little is known about the chemical and sensory characteristics of natural sweet wines obtained by different grape dehydration processes. The main aim of this work is to characterise several natural sweet wines, in order to understand the influence of grape dehydration on the chemical and sensory profile of those wines. First, conventional oenological parameters and low molecular weight phenolic compounds have been determined. Next, sensory descriptive analysis was performed on individual samples based on citation frequencies for aroma attributes and conventional intensity scores for taste and mouth-feel properties. Low molecular weight phenolic compounds and acidity were found in a lower concentration in most wines from off-vine dried grapes. Late harvest wine presented higher amounts of phenolics. White wines showed higher sensory and chemical acidity. Most wines obtained from off-vine and on-vine grape dehydration presented common notes of dry fruits and raisins as aroma properties. Chemical and sensory analyses performed in this study were able to define sweet wines. No significant differences have been found in chemical and sensory profiles of sweet wines according to dehydration processes of the grapes. Late harvest wine and white wines were differentiated from other wines through chemical and sensory analysis.

\section{RESUMO}

Pouco se sabe sobre as características químicas e sensoriais dos vinhos doces naturais obtidos por diferentes processos de desidratação de uvas. O objetivo principal deste trabalho é caracterizar vários vinhos doces naturais, a fim de compreender a influência da desidratação nos perfis químico e sensorial dos referidos vinhos. Em primeiro lugar, foram determinados parâmetros enológicos convencionais e compostos fenólicos de massa molecular baixa. Em seguida, realizou-se uma análise sensorial descritiva baseada em frequências de citação para atributos aromáticos e pontuação de intensidade convencional para o gosto e propriedades táteis na boca. Na maioria dos vinhos de uvas secadas fora da videira, foram encontradas menores concentrações de compostos fenólicos e acidez. Os vinhos de colheita tardia apresentaram maiores quantidades de compostos fenólicos. Os vinhos de variedades brancas mostraram maior acidez química e sensorial. A maioria dos vinhos de uvas desidratadas na videira e fora dela foram caracterizados por notas comuns de frutos secos e passas como descritores aromáticos. As análises químicas e sensoriais realizadas neste trabalho permitiram definir os vinhos doces em estudo. Não foram encontradas diferenças significativas nos perfis químicos e sensoriais dos vinhos doces de acordo com os diferentes processos de desidratação das uvas. Vinhos brancos e vinho de colheita tardia foram discriminados dos outros vinhos através de análises químicas e sensoriais.

Key words: sweet wines, grape dehydration, chemical composition, sensory analysis.

Palavras-chave: vinhos doces, desidratação de uvas, composição química, análise sensorial.

This is an Open Access article distributed under the terms of the Creative Commons Attribution License (http://creativecommons.org/licenses/by/4.0), which permits unrestricted use, distribution, and reproduction in any medium, provided the original work is properly cited. 


\section{INTRODUCTION}

The first wines obtained by human beings were sweet wines due to the climatic conditions where grapes were harvested and processed, which did not allow to finish fermentation. In the Mediterranean basin, the climatic conditions were favorable to leave the grapes in the vine to overripe, or to collect the clusters and allow them to dry in the sun and wind. Throughout history, it has been important to preserve the sweetness in wine, and sweet wines were highly prized in ancient Rome and in the Middle Ages (Scienza, 2013). Nowadays, sweet wines constitute a very small percentage of the world wine trade; however, there is a growing interest in high quality sweet wines. The wine industry tries to diversify the wine market by producing wines with distinctive characteristics and creating new products of high quality. In this regard, the production of sweet wines could be a good way to achieve this goal.

In the production of natural sweet wines (without addition of ethanol), the sugar content can be increased by different techniques. The most common techniques are (Reboredo-Rodríguez et al., 2015):

- On-vine: late harvest wines, noble rot wines (Sauternes, Tokaj), icewines (the grapes are allowed to freeze on the vine in the winter frost season, once harvested they are pressed and the must is concentrated because the water is retained as ice). Due to the difficult climatic conditions needed to produce freezing in certain regions, freezing grapes by technical means (freezing chamber, liquid nitrogen) is used to produce non-authentic (artificial) icewines. The International Organisation of Vine and Wine (OIV) set definition and prescriptions for authentic icewine. Harvesting and pressing should be performed at a recommended temperature lower or equal to $-7{ }^{\circ} \mathrm{C}$. The potential alcohol strength by volume for musts cannot be increased and should be as a minimum $15 \%$ volume (corresponding 110 ${ }^{\circ}$ Oechsle or $25.3{ }^{\circ}$ Brix). The minimal alcoholic strength acquired should be $5.5 \%$ by volume. The maximum limit of volatile acidity should be $(2.1 \mathrm{~g} / \mathrm{L}$ expressed in acetic acid). All grapes used in icewine should come from the same region (OIV, 2018). Hermann (2014) has developed a method for icewine authentication.

- Off-vine: Post harvest dehydration is carried out when grapes are shrinking and losing water. There are several methods such us sun drying (Malaga wines), drying in closed environments (Supurao, Passito, Tostado de Ribeiro), where grapes are dried in a chamber under artificially controlled conditions of temperature, airflow and humidity.
During grape dehydration, in addition to sugar concentration, phenolic and aromatic compounds are concentrated or new compounds are produced (Moreno et al., 2008). Phenolic compounds are abundant in grapes and play an important role in wine quality. Among the non-coloured phenolic compounds, phenolic acids, flavanols and flavonols represent an important group of secondary metabolites in grapes that play an essential role in certain sensory characteristics of wines such as astringency and bitterness (Fischer and Noble, 1994; Vidal et al., 2004). The phenolic profile of the wine varies according to grape variety and degree of ripening, cultural practices, vineyard location and processing and storage techniques (Ramos et al., 1999; Fang et al., 2008). For example, in ice wines, some authors have determined that phenolic acids and flavanols are the major phenolic compounds (Tian et al., 2009a; Tang et al., 2013). Phenolic composition has been studied in icewines from various regions or cultivars (Li et al., 2016; Luo et al., 2017). Kilmartin et al. (2007) observed the impact of freeze concentration and harvest time upon polyphenol composition and concentration. The variations of phenolic compounds in the making of sweet wines has been determined as a function of environmental conditions employed for the dehydration process and of the rate of water loss (Serratosa et al., 2011; Figueiredo-González et al., 2013).

Moreover, aroma is one of the most important quality factors that determines the characteristics of products. The aroma in wine consists of hundred compounds derived from grapes. Due to grape dehydration, either on vine or once harvested, changes in volatile composition of grapes are produced, which means also changes in aromatic profiles of obtained sweet wines (D'Onofrio, 2013; Reboredo-Rodríguez et al., 2015). The mechanisms by which flavours appear in sweet wines are not very clear. However, some studies have pointed out that variety, origin, addition and fermentation management (yeast metabolism in a high sugar concentration medium) influence sensory characteristics (Bellincontro et al., 2004; Budic-Leto et al., 2010; González-Álvarez et al., 2013). The effect of yeast strain in aroma compounds has been also studied (Synos et al., 2015). Crandles et al. (2015) observed that yeast effects depended upon cultivar and vintage. Changes in aroma profile throughout on-vine over-ripening and freezing processes have been also studied (Bowen et al., 2016; Lan et al., 2016). Sensory analysis techniques have been applied to wines during the last decades and have become a common practice to obtain objective characterisation and discrimination of products as well as a tool for quality control (Sáenz-Navajas et al., 2011). 
Few studies have been published about the effect of techniques of grapes dehydration on the chemical composition and sensory characteristics of obtained sweet wines. Therefore, the main aim of this work is the chemical and sensorial characterisation of different sweet wines obtained by different dehydration techniques, in order to compare and distinguish them, and thus to understand the influence of dehydration processes of grapes. In a previous work, a general picture of the grouping of samples (sweet wines under study) in a multidimensional scaling space derived from a sensory sorting task was presented (Rosáenz-Oroz et al., 2012). Thus, a larger scale study was carried out in order to obtain accurate descriptions of those sweet wines and differences among them.

\section{MATERIAL AND METHODS}

\section{Wine Samples}

Ten sweet wines were selected in order to check out different origins, vintages, varieties and processing techniques. Table I shows codes and general information of studied wines. Winemaking conditions of selected wines are detailed below according to the commercial information provided by the wineries.

TABLE I

Wine Samples

Amostras de vinho

\begin{tabular}{|c|c|c|c|c|c|}
\hline RMV1 & RMV1 & Natural icewine & La Rioja (Spain) & Tempranillo (red) & 2011 \\
\hline Malizia & MAL & Natural icewine & La Rioja (Spain) & Tempranillo, Grenache (red) & 2009 \\
\hline No Phone & NOPHO & Late harvest & La Rioja (Spain) & $\begin{array}{c}\text { Tempranillo (red),Viura (white), } \\
\text { Cagazal (white) }\end{array}$ & 2008 \\
\hline Vi de Glass & GLASSGEW & Artificial icewine & Penedés (Spain) & Gewürztraminer (white) & 2010 \\
\hline Verdil de Gel & VERD & Artificial icewine & Alforins (Spain) & Verdil (white) & 2010 \\
\hline Blue Nun & BLUE & Natural icewine & $\begin{array}{c}\text { Rheinhessen } \\
\text { (Germany) }\end{array}$ & Riesling (white) & 2009 \\
\hline Ojuel & OJU & Supurao & La Rioja (Spain) & Tempranillo, Grenache (red) & 2010 \\
\hline
\end{tabular}

RMV and RMV2: grapes were harvested at optimal moment of ripeness (October) and were then frozen in a freezer chamber at $-18{ }^{\circ} \mathrm{C}$. Grapes at a temperature of $-9{ }^{\circ} \mathrm{C}$ were pressed in January. Must with $35^{\circ}$ Brix was fermented in a stainless steel tank at low temperature by selected yeasts.

RMV1: grapes were left on vines until February when cold weather conditions and frosts in vineyard took place. Grapes were collected and pressed immediately in vineyard at a temperature of $-4{ }^{\circ} \mathrm{C}$. Must with 32 ${ }^{\circ}$ Brix was fermented in stainless steel tank at low temperature by selected yeasts.

Ojuel: after the harvest in October, clusters were hung in a high place with natural ventilation, where they would dry until January. The sweet must extracted from those bunches fermented in an oak barrel in the course of a year.

Blue Nun: after grapes that were being picked at -7 ${ }^{\circ} \mathrm{C}$ were pressed and then the juice slowly fermented.
Malizia: after picking grapes at $-8^{\circ} \mathrm{C}$ they were pressed immediately in vineyard, fermentation took place in oak barrels, followed by aging in American oak barrels for six months.

No Phone: dried grapes on vines were harvested in December and then were pressed. Obtained juice fermented in oak barrel.

Verdil de Gel: grapes were harvested with $13 \%$ Probable Alcohol Degree in October. They were introduced in a cold chamber at $-10{ }^{\circ} \mathrm{C}$ for 15 days. Frozen grapes were pressed and the obtained must fermented.

Vi de glass (Riesling and Gewürztraminer): these wines were made with a prototype fed by two freezing chambers which worked with liquid nitrogen at $-15^{\circ} \mathrm{C}$. The must fermented in stainless steel tanks for 2.5 months. 


\section{Chemical Analyses}

Analysis of conventional oenological parameters in wines

The determination of reducing sugars, ethanol content, $\mathrm{pH}$, titratable and volatile acidities were determined by Infrared Spectrometry with Fourier Transformation with a WineScan ${ }^{\mathrm{TM}}$ FT 120 (FOSS ${ }^{\circledR}$, Barcelona, Spain), which was previously calibrated with the official OIV methods (OIV, 2016). The scanning interval set from 930 to $5011 / \mathrm{cm}$. Table II shows the obtained slope and intercept adjustments. Total Polyphenol Index (TPI) was estimated as absorbance at $280 \mathrm{~nm}$ multiplied by 100 (Ribéreau Gayon et al., 2006). L-lactic and L-malic acids were determined by enzymatic methods according to the official methods of AOAC (AOAC, 2016). The analyses were performed in triplicate.

TABLE II

Slope and intercept for FTIR Winescan ${ }^{\mathrm{TM}}$ parameters

Declive e ordenada na origen para parámetros de FTIR Winescan $^{\mathrm{TM}}$

\begin{tabular}{lccc}
\hline Parameter & Slope & Intercept & $\mathbf{R}^{2}$ \\
\hline $\begin{array}{l}\text { \% ethanol (v/V) } \\
\text { Titratable acidity }(\mathrm{g} / \mathrm{L}\end{array}$ & 1.0060 & -0.1832 & 0.9900 \\
tartaric acid) & 0.9633 & 0.1028 & 0.9934 \\
$\mathrm{pH}$ & 1.0197 & -0.0689 & 0.9945 \\
$\begin{array}{l}\text { Volatile acidity }(\mathrm{g} / \mathrm{L} \\
\text { acetic acid) }\end{array}$ & 0.9301 & 0.0182 & 0.9908 \\
Reducing sugars $(\mathrm{g} / \mathrm{L})$ & 1.0359 & -0.1562 & 0.9903 \\
\hline
\end{tabular}

Quantification of low-molecular weight phenolics by UPLC-MS

Flavonols, flavanols and phenolic acids were identified and quantified by UPLC-MS following the method developed by Gonzalez-Hernandez et al. (2014). Analyses were carried out with a Waters Acquity Ultra Performance LC system (Milford, MA, USA) coupled to a Microtof-Q (Q-TOF) mass spectrometer from Bruker Daltonik (GMBH, Germany) equipped with an Apollo II ESI (electrospray) source and controlled by Bruker Daltonics DataAnalysis software. Wine samples were previously filtered with $0.22 \mu \mathrm{m}$ nylon discs and injected directly. UPLC separation was achieved using an acquity BEH C18 column $(100 \mathrm{~mm} \times 2.1$ mm, i.d., $1.7 \mu \mathrm{m}$ particle size, Waters), kept at $40{ }^{\circ} \mathrm{C}$. Mobile phase flow rate was $0.45 \mathrm{~mL} / \mathrm{min}$ and the injection volume was $7.5 \mu \mathrm{L}$. Solvents were (A) water/formic acid $(0.1 \%)$, and (B) acetonitrile/formic acid $(0.1 \%)$. The gradient programme employed was as follows: $0-4 \mathrm{~min}, 99-92 \% \mathrm{~A} ; 4-11 \mathrm{~min}, 92-70 \%$ A; 11-13.5 min, 70-0\% A; $13.5-14.5 \mathrm{~min}, 0-99 \%$ A.
The ESI source was operated in negative mode recorded in the range of $\mathrm{m} / \mathrm{z} 120$ and 1500 . The optimised conditions of the ESI source were as follows: capillary voltage, $3.5 \mathrm{kV}$; ESI source temperature, $180{ }^{\circ} \mathrm{C}$; desolvation temperature, 200 ${ }^{\circ} \mathrm{C}$; cone gas flow, $9 \mathrm{~L} / \mathrm{min}$; the nebulizer gas was set at 3 bar and $25{ }^{\circ} \mathrm{C}$. The identity assignation of compounds was carried out by comparison of their retention time $\left(t_{\mathrm{R}}\right), \mathrm{MS}$ and MS/MS spectra with those of their respective commercially available standards. Compounds with no available standards were identified by their MS/MS spectrum compared to the retention time and spectral mass from the literature (Schwarz et al., 2009; Sáenz-Navajas et al., 2010a). The quantification was expressed in $\mathrm{mg} / \mathrm{L}$ of caffeic acid, catechin and quercetin (Sigma-Aldrich) for phenolic acids, flavanols and flavonols, respectively. The analyses were performed in triplicate. Table III shows the phenolic compounds identified by this method in wine samples.

\section{Sensory analysis}

Sensory analysis methodology applied in this work was developed by other authors (Sáenz-Navajas et al., 2011). A total of 29 students, wine consumers and members of the University of La Rioja (16 female and 13 male from 21 to 57 years old, average age 29 years) were recruited based on their interests and availability to conduct a session of one hour per week for 8 weeks. All judges were interested in the world of wine, with experience in similar studies of sensory analysis.

Panellists attended eight descriptive sensory training sessions (about 1 hour per session) over a two-month period, during which panellists worked in subgroups led by the same leader and following the same guidelines. They were given a list of 113 terms (including 94 specific terms and 19 more general terms) obtained from the literature (Campo et al., 2008; Sáenz-Navajas et al., 2011). During the training, different reference standards representative of aroma, taste and astringency were presented. Aroma standards were mainly natural products (fruits, juices, spices, vegetables, among others) prepared at the beginning of each session, or odorants supplied by Sentosphère (Paris, France), Firmenich (Geneva, Switzerland) and "Le Nez du Vin "(Jean Lenoir, Provence, France). For taste and astringency training, solutions with different concentrations of sugar (0$200 \mathrm{~g} / \mathrm{L})$ for sweetness, tartaric acid $(0-2 \mathrm{~g} / \mathrm{L})$ for acidity, quinine sulfate $(0-10 \mathrm{mg} / \mathrm{L})$ for bitterness, and potassium and aluminum sulphate $(0-5 \mathrm{~g} / \mathrm{L})$ for astringency were presented to the panel. These solutions aid to recognition and discrimination between different oral sensations. 
TABLE III

Low-molecular weight phenolics found by UPLC-MS

Compostos fenólicos de massa molecular baixa detetados por UPLC-MS

\begin{tabular}{|c|c|c|c|c|}
\hline $\begin{array}{l}\text { Retention time } \\
\quad\left(t_{R}\right)(\mathrm{min})\end{array}$ & Standard & {$[\mathbf{M}-\mathbf{H}]^{-}(\mathbf{m} / \mathbf{z})$} & Qualifier ion (m/z) & Molecular Formula \\
\hline \multicolumn{5}{|l|}{ Acids and derivatives } \\
\hline 0.71 & cis-aconitic acid & 173.00 & 129 & $\mathrm{C}_{6} \mathrm{H}_{5} \mathrm{O}_{6}$ \\
\hline 0.86 & trans-aconitic acid & 193.00 & 129 & $\mathrm{C}_{6} \mathrm{H}_{5} \mathrm{O}_{6}$ \\
\hline 1.33 & gallic acid & 169.01 & 125 & $\mathrm{C}_{7} \mathrm{H}_{5} \mathrm{O}_{5}$ \\
\hline 2.08 & protocatechuic acid & 153.01 & 109 & $\mathrm{C}_{7} \mathrm{H}_{5} \mathrm{O}_{4}$ \\
\hline 3.49 & vanillic acid & 167.03 & 152 & $\mathrm{C}_{9} \mathrm{H}_{9} \mathrm{O}_{5}$ \\
\hline 4.24 & syringic acid & 197.04 & 182 & $\mathrm{C}_{9} \mathrm{H}_{10} \mathrm{O}_{5}$ \\
\hline 8.18 & protocatechuic acid ethyl ester & 181.04 & 153 & $\mathrm{C}_{9} \mathrm{H}_{9} \mathrm{O}_{4}$ \\
\hline 8.47 & cis-resveratrol & 227.07 & 143 & $\mathrm{C}_{14} \mathrm{H}_{11} \mathrm{O}_{3}$ \\
\hline \multicolumn{5}{|c|}{ Hydroxycinnamic acids and derivatives } \\
\hline 2.17 & cis-caftaric acid & 311.04 & 179 & $\mathrm{C}_{13} \mathrm{H}_{12} \mathrm{O}_{9}$ \\
\hline 2.22 & trans-caftaric acid & 311.02 & 179 & $\mathrm{C}_{13} \mathrm{H}_{12} \mathrm{O}_{9}$ \\
\hline 3.03 & coutaric acid & 295.04 & 119 & $\mathrm{C}_{13} \mathrm{H}_{12} \mathrm{O}_{8}$ \\
\hline 3.52 & caffeic acid & 179.03 & 135 & $\mathrm{C}_{9} \mathrm{H}_{7} \mathrm{O}_{4}$ \\
\hline 3.68 & ferulic acid & 193.04 & 178 & $\mathrm{C}_{10} \mathrm{H}_{10} \mathrm{O}_{4}$ \\
\hline 5.74 & cinnamic acid & 148.16 & 103 & $\mathrm{C}_{9} \mathrm{H}_{8} \mathrm{O}_{2}$ \\
\hline 5.36 & cis-coumaric acid & 163.03 & 119 & $\mathrm{C}_{9} \mathrm{H}_{7} \mathrm{O}_{3}$ \\
\hline 6.15 & trans-coumaric acid & 163.03 & 119 & $\mathrm{C}_{9} \mathrm{H}_{7} \mathrm{O}_{3}$ \\
\hline 10.03 & Caffeic acid ethyl ester & 207.21 & 179 & $\mathrm{C}_{11} \mathrm{H}_{12} \mathrm{O}_{4}$ \\
\hline \multicolumn{5}{|l|}{ flavanols } \\
\hline 2.74 & epigallocatechin & 305.06 & 203 & $\mathrm{C}_{15} \mathrm{H}_{13} \mathrm{O}_{7}$ \\
\hline 3.12 & catechin & 289.07 & 203 & $\mathrm{C}_{15} \mathrm{H}_{13} \mathrm{O}_{6}$ \\
\hline 4.73 & epicatechin & 289.07 & 203 & $\mathrm{C}_{15} \mathrm{H}_{13} \mathrm{O}_{6}$ \\
\hline \multicolumn{5}{|l|}{ flavonols } \\
\hline 5.09 & myricetin-3-O-glucoside & 449.11 & 317 & $\mathrm{C}_{21} \mathrm{H}_{19} \mathrm{O}_{12}$ \\
\hline 8.10 & kaempferol-3-O-glucoside & 447.08 & 287 & $\mathrm{C}_{21} \mathrm{H}_{19} \mathrm{O}_{11}$ \\
\hline
\end{tabular}

The training period comprised of two phases: a general phase and a specific phase of the type of product. During the general training phase (six sessions), the panellists became familiar with the attributes of the aroma and with intensity rating of sweetness, acidity, bitterness, astringency, aromatic intensity and global intensity as well as persistence. Then, they had to evaluate three to five different wines by describing their odour properties (orthonasally) by choosing up to five descriptors from the list and by rating tastes and astringency on a 10point scale $(0=$ "absence," $1=$ "very low" and $9=$ "very high"), global intensity on a nine-point scale (1 = "very low" and 9 = "very high") and global persistence on a nine-point scale $(1=$ "very short" and 9 = "very long"). The session ended with a discussion during which the panel leader compared the aroma descriptors and the taste intensity scores given by panellists to describe each wine. The wines selected for this training phase had intense and easily recognisable flavour and astringency properties, including red, white and rosé wines of various varieties and origins.

The specific training phase consisted of two sessions during which the panellists became familiar with the type of samples of the study. During this phase, panellists described the aromatic properties by choosing up to five terms from the list provided, and rated the intensity of sweetness, acidity, bitterness, astringency, aromatic intensity and global intensity, as well as persistence of commercial sweet wines.

In the evaluation of the wines, each panellist took part in a 45-minute session. $15 \mathrm{~mL}$ wine samples were presented in dark wine glasses approved by ISO (ISO, 1977) and coded with three-digit random numbers and arranged in random order. Samples were served at room temperature and covered with plastic Petri dishes. Panellists evaluated wines in separate individual booths. They first had to evaluate each wine orthonasally and rating the aromatic intensity and then describe the aroma by choosing a maximum of five attributes from the list of aromatic terms according to frequency citation method (Campo et al., 2008). Then, they were asked to rate sweetness, acidity, bitterness, astringency, global intensity and persistence of each sample using the abovementioned structured scales for each wine. Mineral water and unsalted crackers were provided for cleansing palate and rinsing between each sample. The sessions were held in a ventilated and airconditioned sensory analysis room (about $20^{\circ} \mathrm{C}$ ).

\section{Data Analysis}

\section{Analysis of panel performance}

In order to assess panel performance, an average reproducibility index $\left(\mathrm{R}_{\mathrm{i}}\right)$ was calculated for each panellist according to the method proposed by Campo et al. (2008). A value of $\mathrm{R}_{\mathrm{i}}$ greater than 0.2 was 
established to consider the judge's data. As for the attributes evaluated in mouth and aromatic intensity, a principal component analysis (PCA) was performed for each attribute. From the panellist's projections in the loading plot of the PCA, the agreement (grouped projections) and disagreement (spread projections) of panellists were evaluated. For each attribute, panellists' data that were spread were not considered. According to the $\mathrm{R}_{\mathrm{i}}$ and the PCA projections, the data of 22 panellists were considered for the statistical treatment of the data.

\section{Product characterisation}

Citation frequency of aromatic terms was calculated. Only the descriptors cited by a minimum of five judges (15\% of the panel) in at least one of the wines were considered for subsequent statistical analysis. From these terms, those with citation frequencies higher than those expected by chance were chosen according to the chi-square distribution. Thus, 13 significant terms were considered $(\mathrm{p}<0.05)$.

A one-way ANOVA (Analysis of Variance) was carried out on chemical analysis. A two-way ANOVA with judges as a block factor was performed on scores derived from in-mouth attributes and aromatic intensity. Significant differences were determined through Tukey test $(\mathrm{p}<0.05)$.

Data derived from chemical analyses and descriptive sensory analysis were analysed using Principal Component Analysis (PCA) and Correspondence Analysis (CA). PCA was performed on the average ratings over the judges for in-mouth attributes sweetness, acidity, bitterness, astringency, global intensity and persistence and for each wine (correlation matrix) while CA was performed on the contingency table containing the mean citation frequencies of significant aroma descriptors. In order to choose the number of principal components that should be retained, dimensions with eigenvalue higher than the average eigenvalue (Kaiser's law) were used for both PCA and CA spaces.

Finally, a hierarchical cluster analysis (HCA) with the Ward criteria was applied to the factorial coordinates of the wines in the spaces defined by the PCA and CA according to these parameters. The clusters identified from the tree diagram were consolidated by aggregation around their mobile centres. In both cases, the terms that best characterised each of the clusters were identified by their probability $\mathrm{p}<0.05$. (Morineau et al., 1995).

All analyses were performed with IBM SPSS Statistics program (Version 20.0, SPSS Inc., Illinois, USA).

\section{RESULTS AND DISCUSSION}

\section{Chemical Characterisation}

Average values of conventional oenological parameters and low molecular weight phenolic compounds determined in wine samples are shown in Tables IV and V. The calculated ANOVA indicates that there are significant differences between wines ( $\mathrm{p}$ $<0.05$ ) for each analysed variable.

In order to simplify the results presentation and their discussion, phenolic compounds have been grouped into four different categories: acids and derivatives, hydroxycinnamic acids, flavanols and flavonols, as shown in Table III.

TABLE IV

Conventional oenological parameters of wine samples

Parâmetros enológicos convencionais de amostras de vinho

\begin{tabular}{|c|c|c|c|c|c|c|c|c|}
\hline Samples & $\begin{array}{c}\text { \% Ethanol } \\
\text { (v/V) }\end{array}$ & $\begin{array}{c}\text { Titratable } \\
\text { acidity (g/L } \\
\text { tartaric acid) }\end{array}$ & pH & $\begin{array}{c}\text { Volatile } \\
\text { acidity } \\
\text { (g/L acetic } \\
\text { acid) }\end{array}$ & $\begin{array}{l}\text { Lactic acid } \\
\qquad(\mathrm{g} / \mathrm{L})\end{array}$ & $\begin{array}{c}\text { Malic acid } \\
\text { (g/L) }\end{array}$ & TPI & $\begin{array}{c}\text { Reducing } \\
\text { sugars }(g / L)\end{array}$ \\
\hline RMV & $10.27 \pm 0.01^{\mathrm{d}}$ & $5.43 \pm 0.02^{c}$ & $4.05 \pm 0.01^{\mathrm{f}}$ & $1.19 \pm 0.01^{\mathrm{f}}$ & $0.08 \pm 0.01^{\mathrm{a}}$ & $2.70 \pm 0.03^{\mathrm{d}}$ & $34.98 \pm 0.05^{g}$ & $200.89 \pm 1.55^{\mathrm{h}}$ \\
\hline RMV1 & $10.87 \pm 0.01^{\mathrm{g}}$ & $4.89 \pm 0.01^{\mathrm{b}}$ & $4.03 \pm 0.01^{\mathrm{f}}$ & $0.70 \pm 0.01^{\text {bc }}$ & $0.09 \pm 0.01^{\mathrm{ab}}$ & $2.85 \pm 0.06^{\mathrm{de}}$ & $26.36 \pm 0.02^{\mathrm{e}}$ & $150.41 \pm 0.43^{\mathrm{e}}$ \\
\hline RMV2 & $11.61 \pm 0.01^{\mathrm{h}}$ & $5.46 \pm 0.02^{\mathrm{c}}$ & $3.47 \pm 0.01^{\mathrm{c}}$ & $0.72 \pm 0.01^{\text {bc }}$ & $0.17 \pm 0.01^{\mathrm{b}}$ & $3.08 \pm 0.06^{e f}$ & $38.23 \pm 0.05^{\mathrm{h}}$ & $162.66 \pm 0.22^{f}$ \\
\hline MAL & $12.63 \pm 0.01^{\mathrm{i}}$ & $7.07 \pm 0.02^{\mathrm{e}}$ & $3.63 \pm 0.02^{\mathrm{c}}$ & $0.94 \pm 0.03^{\mathrm{d}}$ & $0.56 \pm 0.02^{\mathrm{e}}$ & $1.88 \pm 0.15^{\mathrm{bc}}$ & $28.38 \pm 0.02^{f}$ & $144.39 \pm 1.18^{c}$ \\
\hline NOPHO & $14.74 \pm 0.01^{\mathrm{j}}$ & $4.64 \pm 0.02^{\mathrm{a}}$ & $2.81 \pm 0.01^{\mathrm{e}}$ & $0.57 \pm 0.01^{\mathrm{a}}$ & $0.59 \pm 0.03^{\mathrm{e}}$ & $1.34 \pm 0.06^{\mathrm{a}}$ & $21.44 \pm 0.02^{\mathrm{c}}$ & $70.68 \pm 0.32^{\mathrm{a}}$ \\
\hline GLASSRIES & $10.45 \pm 0.01^{\mathrm{f}}$ & $8.99 \pm 0.01^{\mathrm{h}}$ & $2.81 \pm 0.02^{\mathrm{a}}$ & $0.73 \pm 0.02^{\mathrm{c}}$ & $0.04 \pm 0.01^{\mathrm{a}}$ & $2.24 \pm 0.14^{\mathrm{c}}$ & $19.97 \pm 0.02^{\mathrm{b}}$ & $148.72 \pm 0.93^{\mathrm{de}}$ \\
\hline GLASSGEW & $10.31 \pm 0.01^{\mathrm{e}}$ & $8.77 \pm 0.03^{g}$ & $2.86 \pm 0.01^{\mathrm{a}}$ & $0.69 \pm 0.01^{\mathrm{bc}}$ & $0.05 \pm 0.01^{\mathrm{a}}$ & $2.02 \pm 0.08^{\mathrm{bc}}$ & $20.29 \pm 0.14^{b}$ & $146.75 \pm 0.77^{\mathrm{cd}}$ \\
\hline VERD & $9.78 \pm 0.01^{\mathrm{b}}$ & $8.03 \pm 0.01^{\mathrm{f}}$ & $3.20 \pm 0.01^{\mathrm{b}}$ & $0.65 \pm 0.02^{\mathrm{b}}$ & $0.02 \pm 0.01^{\mathrm{a}}$ & $3.34 \pm 0.04^{\mathrm{f}}$ & $39.28 \pm 0.22^{\mathrm{i}}$ & $135.09 \pm 0.12^{\mathrm{b}}$ \\
\hline BLUE & $8.50 \pm 0.01^{\mathrm{a}}$ & $9.10 \pm 0.01^{\mathrm{i}}$ & $3.51 \pm 0.01^{\mathrm{cd}}$ & $1.01 \pm 0.01^{\mathrm{de}}$ & $0.44 \pm 0.02^{\mathrm{d}}$ & $4.04 \pm 0.03^{g}$ & $11.05 \pm 0.02^{\mathrm{a}}$ & $172.53 \pm 0.20^{\mathrm{g}}$ \\
\hline OJU & $10.21 \pm 0.02^{\mathrm{c}}$ & $6.31 \pm 0.01^{\mathrm{d}}$ & $3.56 \pm 0.02^{\mathrm{d}}$ & $1.06 \pm 0.04^{\mathrm{e}}$ & $0.29 \pm 0.03^{c}$ & $1.69 \pm 0.13^{\mathrm{ab}}$ & $23.59 \pm 0.12^{\mathrm{d}}$ & $233.62 \pm 0.57^{\mathrm{i}}$ \\
\hline
\end{tabular}

Means followed by different letters in a column are statistically different ( $\mathrm{p}<0.05)$. 
TABLE V

Phenolic compounds concentrations (mg/L) in wine samples

Concentrações de compostos fenólicos $(\mathrm{mg} / \mathrm{L})$ em amostras de vinho

\begin{tabular}{|c|c|c|c|c|c|c|c|c|c|c|}
\hline & RMV & RMV1 & RMV2 & MAL & NOPHO & GLASSRIES & GLASGEW & VERD & BLUE & OJU \\
\hline \multicolumn{11}{|l|}{$\begin{array}{l}\text { Acids and } \\
\text { derivatives }\end{array}$} \\
\hline cis-aconitic acid & $2.28 \pm 0.06^{\mathrm{d}}$ & $2.64 \pm 0.08^{\mathrm{e}}$ & $1.64 \pm 0.12^{c}$ & $2.29 \pm 0.14^{\mathrm{d}}$ & $1.99 \pm 0.07^{\mathrm{d}}$ & $0.60 \pm 0.01^{\mathrm{a}}$ & $0.94 \pm 0.05^{\mathrm{b}}$ & $1.45 \pm 0.04^{\mathrm{c}}$ & $1.39 \pm 0.06^{\mathrm{c}}$ & $1.99 \pm 0.07^{\mathrm{d}}$ \\
\hline trans-aconitic acid & $0.66 \pm 0.03^{b c}$ & $0.51 \pm 0.02^{\mathrm{abc}}$ & $0.69 \pm 0.01^{\mathrm{cd}}$ & $1.08 \pm 0.12^{\mathrm{e}}$ & $0.64 \pm 0.02^{\mathrm{bc}}$ & $0.57 \pm 0.01^{\mathrm{bc}}$ & $0.50 \pm 0.01^{\mathrm{ab}}$ & $0.37 \pm 0.02^{\mathrm{a}}$ & $0.66 \pm 0.01^{b c}$ & $0.86 \pm 0.01^{\mathrm{d}}$ \\
\hline Gallic acid & $0.94 \pm 0.01^{c}$ & $1.84 \pm 0.02^{\mathrm{d}}$ & $1.24 \pm 0.11^{c}$ & $1.69 \pm 0.21^{\mathrm{d}}$ & $5.36 \pm 0.10^{f}$ & $0.09 \pm 0.01^{\mathrm{a}}$ & $0.11 \pm 0.02^{\mathrm{a}}$ & $0.52 \pm 0.01^{b}$ & $0.02 \pm 0.00^{\mathrm{a}}$ & $2.91 \pm 0.09^{\mathrm{e}}$ \\
\hline protocatechuic acid & $0.12 \pm 0.01^{\mathrm{a}}$ & $1.42 \pm 0.02^{\mathrm{g}}$ & $0.13 \pm 0.01^{\mathrm{a}}$ & $0.54 \pm 0.01^{\mathrm{d}}$ & $0.61 \pm 0.01^{\mathrm{e}}$ & $0.09 \pm 0.01^{\mathrm{a}}$ & $0.22 \pm 0.01^{\mathrm{b}}$ & $0.26 \pm 0.01^{\mathrm{b}}$ & $0.41 \pm 0.01^{c}$ & $1.03 \pm 0.01^{\mathrm{f}}$ \\
\hline vanillic acid & $0.07 \pm 0.01^{\mathrm{d}}$ & $0.15 \pm 0.01^{\mathrm{e}}$ & $0.01 \pm 0.00^{\mathrm{ab}}$ & $0.02 \pm 0.00^{\mathrm{b}}$ & $0.05 \pm 0.01^{c}$ & $\mathrm{nd}^{\mathrm{a}}$ & $\mathrm{nd}^{\mathrm{a}}$ & $\mathrm{nd}^{\mathrm{a}}$ & $\mathrm{nd}^{\mathrm{a}}$ & $0.03 \pm 0.01^{\mathrm{b}}$ \\
\hline syringic acid & $0.25 \pm 0.04^{\mathrm{b}}$ & $1.41 \pm 0.06^{c}$ & $0.01 \pm 0.00^{\mathrm{a}}$ & $0.04 \pm 0.01^{\mathrm{a}}$ & $0.02 \pm 0.00^{\mathrm{a}}$ & $\mathrm{nd}^{\mathrm{a}}$ & $\mathrm{nd}^{\mathrm{a}}$ & $\mathrm{nd}^{\mathrm{a}}$ & $\mathrm{nd}^{\mathrm{a}}$ & $0.03 \pm 0.01^{\mathrm{a}}$ \\
\hline $\begin{array}{l}\text { protocatechuic acid } \\
\text { ethyl ester }\end{array}$ & $0.39 \pm 0.04^{\text {cd }}$ & $1.13 \pm 0.01^{\mathrm{e}}$ & $0.07 \pm 0.01^{\mathrm{a}}$ & $0.38 \pm 0.02^{\mathrm{cd}}$ & $1.64 \pm 0.06^{f}$ & $0.04 \pm 0.01^{\mathrm{a}}$ & $0.20 \pm 0.01^{\mathrm{b}}$ & $0.31 \pm 0.01^{c}$ & $0.08 \pm 0.01^{\mathrm{a}}$ & $0.48 \pm 0.04^{\mathrm{d}}$ \\
\hline cis-resveratrol & $\mathrm{nd}^{\mathrm{a}}$ & $0.03 \pm 0.00^{\mathrm{d}}$ & $0.01 \pm 0.00^{\mathrm{b}}$ & $\mathrm{nd}^{\mathrm{a}}$ & $0.02 \pm 0.00^{c}$ & $\mathrm{nd}^{\mathrm{a}}$ & $\mathrm{nd}^{\mathrm{a}}$ & $\mathrm{nd}^{\mathrm{a}}$ & $\mathrm{nd}^{\mathrm{a}}$ & $0.02 \pm 0.00^{\mathrm{bc}}$ \\
\hline \multicolumn{11}{|l|}{$\begin{array}{l}\text { Hydroxycinnamic } \\
\text { acids and } \\
\text { derivatives }\end{array}$} \\
\hline cis-caftaric acid & $0.69 \pm 0.06^{\mathrm{a}}$ & $7.51 \pm 1.80^{\mathrm{b}}$ & $\mathrm{nd}^{\mathrm{a}}$ & $\mathrm{nd}^{\mathrm{a}}$ & $\mathrm{nd}^{\mathrm{a}}$ & $0.01 \pm 0.00^{\mathrm{a}}$ & $0.01 \pm 0.00^{\mathrm{a}}$ & $\mathrm{nd}^{\mathrm{a}}$ & $\mathrm{nd}^{\mathrm{a}}$ & $\mathrm{nd}^{\mathrm{a}}$ \\
\hline trans-caftaric acid & $2.37 \pm 0.08^{b}$ & $8.22 \pm 1.46^{c}$ & $\mathrm{nd}^{\mathrm{a}}$ & $\mathrm{nd}^{\mathrm{a}}$ & $\mathrm{nd}^{\mathrm{a}}$ & $0.01 \pm 0.00^{\mathrm{a}}$ & $0.01 \pm 0.00^{\mathrm{a}}$ & $\mathrm{nd}^{\mathrm{a}}$ & $0.01 \pm 0.00^{\mathrm{a}}$ & $\mathrm{nd}^{\mathrm{a}}$ \\
\hline Coutaric acid & $3.23 \pm 0.11^{\mathrm{b}}$ & $14.66 \pm 0.54^{c}$ & $\mathrm{nd}^{\mathrm{a}}$ & $0.01 \pm 0.00^{\mathrm{a}}$ & $\mathrm{nd}^{\mathrm{a}}$ & $0.02 \pm 0.00^{\mathrm{a}}$ & $0.01 \pm 0.00^{\mathrm{a}}$ & $\mathrm{nd}^{\mathrm{a}}$ & $0.10 \pm 0.01^{\mathrm{a}}$ & $0.07 \pm 0.01^{\mathrm{a}}$ \\
\hline Caffeic acid & $2.24 \pm 0.01^{\mathrm{cd}}$ & $1.49 \pm 0.25^{b}$ & $0.76 \pm 0.02^{\mathrm{a}}$ & $2.06 \pm 0.01^{c}$ & $2.64 \pm 0.06^{\mathrm{d}}$ & $2.52 \pm 0.05^{\text {cd }}$ & $2.59 \pm 0.05^{\mathrm{d}}$ & $5.18 \pm 0.07^{\mathrm{e}}$ & $2.43 \pm 0.05^{c d}$ & $6.77 \pm 0.31^{f}$ \\
\hline ferulic acid & $2.21 \pm 0.02^{f}$ & $1.40 \pm 0.19^{\mathrm{e}}$ & $0.47 \pm 0.04^{\mathrm{bcd}}$ & $0.07 \pm 0.01^{\mathrm{a}}$ & $0.27 \pm 0.05^{\mathrm{ab}}$ & $1.61 \pm 0.11^{\mathrm{e}}$ & $0.34 \pm 0.03^{\mathrm{abc}}$ & $0.33 \pm 0.01^{\mathrm{abc}}$ & $0.70 \pm 0.06^{\mathrm{d}}$ & $0.62 \pm 0.01^{\mathrm{cd}}$ \\
\hline cinnamic acid & $0.37 \pm 0.04^{c}$ & $0.70 \pm 0.03^{\mathrm{d}}$ & $0.04 \pm 0.01^{\mathrm{ab}}$ & $0.06 \pm 0.00^{\mathrm{ab}}$ & $0.05 \pm 0.02^{\mathrm{ab}}$ & $0.01 \pm 0.00^{\mathrm{ab}}$ & $0.01 \pm 0.00^{\mathrm{a}}$ & $0.02 \pm 0.01^{\mathrm{ab}}$ & $0.09 \pm 0.01^{\mathrm{b}}$ & $0.09 \pm 0.01^{\mathrm{ab}}$ \\
\hline cis-coumaric acid & $0.05 \pm 0.01^{\mathrm{a}}$ & $1.16 \pm 0.13^{\mathrm{e}}$ & $0.07 \pm 0.02^{\mathrm{a}}$ & $0.30 \pm 0.04^{\mathrm{bc}}$ & $0.67 \pm 0.01^{d}$ & $0.07 \pm 0.01^{\mathrm{a}}$ & $0.03 \pm 0.00^{\mathrm{a}}$ & $0.49 \pm 0.02^{\mathrm{cd}}$ & $0.29 \pm 0.01^{\mathrm{b}}$ & $0.53 \pm 0.02^{\mathrm{d}}$ \\
\hline trans-coumaric acid & $\mathrm{nd}^{\mathrm{a}}$ & $\mathrm{nd}^{\mathrm{a}}$ & $\mathrm{nd}^{\mathrm{a}}$ & $0.06 \pm 0.01^{\mathrm{d}}$ & $0.04 \pm 0.01^{c}$ & $0.02 \pm 0.00^{\mathrm{b}}$ & $0.02 \pm 0.00^{\mathrm{b}}$ & $\mathrm{nd}^{\mathrm{a}}$ & $0.08 \pm 0.01^{\mathrm{e}}$ & $0.07 \pm 0.01^{\mathrm{de}}$ \\
\hline $\begin{array}{l}\text { Caffeic acid ethyl } \\
\text { ester }\end{array}$ & $0.30 \pm 0.01^{\mathrm{a}}$ & $0.56 \pm 0.02^{\mathrm{ab}}$ & $0.21 \pm 0.01^{\mathrm{a}}$ & $3.05 \pm 0.11^{\mathrm{d}}$ & $4.58 \pm 0.12^{\mathrm{e}}$ & $0.61 \pm 0.09^{\mathrm{abc}}$ & $0.94 \pm 0.04^{\mathrm{bc}}$ & $7.40 \pm 0.29^{f}$ & $1.05 \pm 0.04^{c}$ & $6.98 \pm 0.10^{f}$ \\
\hline \multicolumn{11}{|l|}{ Flavanols } \\
\hline Epigallocatechin & $0.02 \pm 0.00^{\mathrm{bc}}$ & $0.03 \pm 0.01^{c}$ & $0.01 \pm 0.00^{\mathrm{b}}$ & $\mathrm{nd}^{\mathrm{a}}$ & $0.02 \pm 0.00^{\mathrm{c}}$ & $\mathrm{nd}^{\mathrm{a}}$ & $\mathrm{nd}^{\mathrm{a}}$ & $\mathrm{nd}^{\mathrm{a}}$ & $\mathrm{nd}^{\mathrm{a}}$ & $\mathrm{nd}^{\mathrm{a}}$ \\
\hline Catechin & $0.16 \pm 0.01^{\mathrm{de}}$ & $0.06 \pm 0.01^{\mathrm{c}}$ & $0.17 \pm 0.03^{\mathrm{e}}$ & $\mathrm{nd}^{\mathrm{a}}$ & $0.13 \pm 0.01^{\mathrm{d}}$ & $0.03 \pm 0.01^{\mathrm{b}}$ & $0.03 \pm 0.00^{\mathrm{bc}}$ & $0.03 \pm 0.01^{\mathrm{bc}}$ & $\mathrm{nd}^{\mathrm{a}}$ & $0.02 \pm 0.00^{\mathrm{ab}}$ \\
\hline Epicatechin & $0.09 \pm 0.02^{\mathrm{ab}}$ & $0.11 \pm 0.02^{\mathrm{b}}$ & $0.01 \pm 0.00^{\mathrm{ab}}$ & $\mathrm{nd}^{\mathrm{a}}$ & $0.02 \pm 0.00^{\mathrm{ab}}$ & $0.01 \pm 0.00^{\mathrm{ab}}$ & $\mathrm{nd}^{\mathrm{a}}$ & $0.01 \pm 0.00^{\mathrm{a}}$ & $\mathrm{nd}^{\mathrm{a}}$ & $0.02 \pm 0.00^{\mathrm{ab}}$ \\
\hline \multicolumn{11}{|l|}{ Flavonols } \\
\hline $\begin{array}{l}\text { Myricetin-3-O- } \\
\text { glucoside }\end{array}$ & $\mathrm{nd}^{\mathrm{a}}$ & $0.02 \pm 0.00^{c}$ & $0.01 \pm 0.00^{\mathrm{b}}$ & $\mathrm{nd}^{\mathrm{a}}$ & $0.02 \pm 0.00^{\mathrm{bc}}$ & $0.02 \pm 0.00^{c}$ & $\mathrm{nd}^{\mathrm{a}}$ & $\mathrm{nd}^{\mathrm{a}}$ & $\mathrm{nd}^{\mathrm{a}}$ & $\mathrm{nd}^{\mathrm{a}}$ \\
\hline $\begin{array}{l}\text { Kaempferol-3-O- } \\
\text { glucoside }\end{array}$ & $\mathrm{nd}^{\mathrm{a}}$ & $\mathrm{nd}^{\mathrm{a}}$ & $\mathrm{nd}^{\mathrm{a}}$ & $\mathrm{nd}^{\mathrm{a}}$ & $\mathrm{nd}^{\mathrm{a}}$ & $\mathrm{nd}^{\mathrm{a}}$ & $\mathrm{nd}^{\mathrm{a}}$ & $0.01 \pm 0.00$ & $\mathrm{nd}^{\mathrm{a}}$ & $\mathrm{nd}^{\mathrm{a}}$ \\
\hline
\end{tabular}

There was a negative correlation between $\mathrm{pH}$ and titratable acidity, as expected $(\mathrm{F}=-0.779, \mathrm{p}=0.088)$ and positive correlation between hydroxybenzoic acids and derivatives $(\mathrm{F}=0.681, \mathrm{p}=0.030)$ and flavanols $(\mathrm{F}=0.669, \mathrm{p}=0.035)$. Volatile acidity is positively correlated with the concentration of sugars $(\mathrm{F}=0.802, \mathrm{p}=0.005)$, which is logical in production of sweet wines (response to yeast osmotic stress), and negatively with flavonols $(F=-0.748, p=0.013)$. Ethanol content is inversely correlated with sugar content $(\mathrm{F}=-0.683, \mathrm{p}=0.030)$ and malic acid $(\mathrm{F}=$ $0.681, p=0.030)$. This fact is due to a lower uptake of sugars in fermentation, malolactic fermentation carried out by some wines or a less fruit ripening state, since less mature grapes present higher concentration of malic acid and a lower probable alcoholic content. There is no correlation between the TPI and phenolic compounds determined, indicating that other groups of phenolic compounds are involved in the determination of TPI.

In order to assess similarities in the studied samples, a PCA was calculated with parameter values in Tables IV and V. Figure 1 shows the two-dimensional plot with the projection of samples and variables. The first dimension accounted for $35.5 \%$ of the total variance and it was mainly characterised positively by the concentration of phenolic acids and derivatives (89\%), ethanol (79\%) and flavanols (71\%) and 
negatively by titratable acidity (92\%). The most alcoholic wines with higher content in phenolic acids and flavanols were located on the right side of the plane, while on the left side more acid wines were placed. PC 2 accounts for almost $24 \%$ of the original variance and it was negatively correlated to sugars (79\%), volatile acidity (76\%) and $\mathrm{pH}$ (71\%). Therefore, wines with high sugar concentration and volatile acidity with less acidity were located in the lower part of the plot.
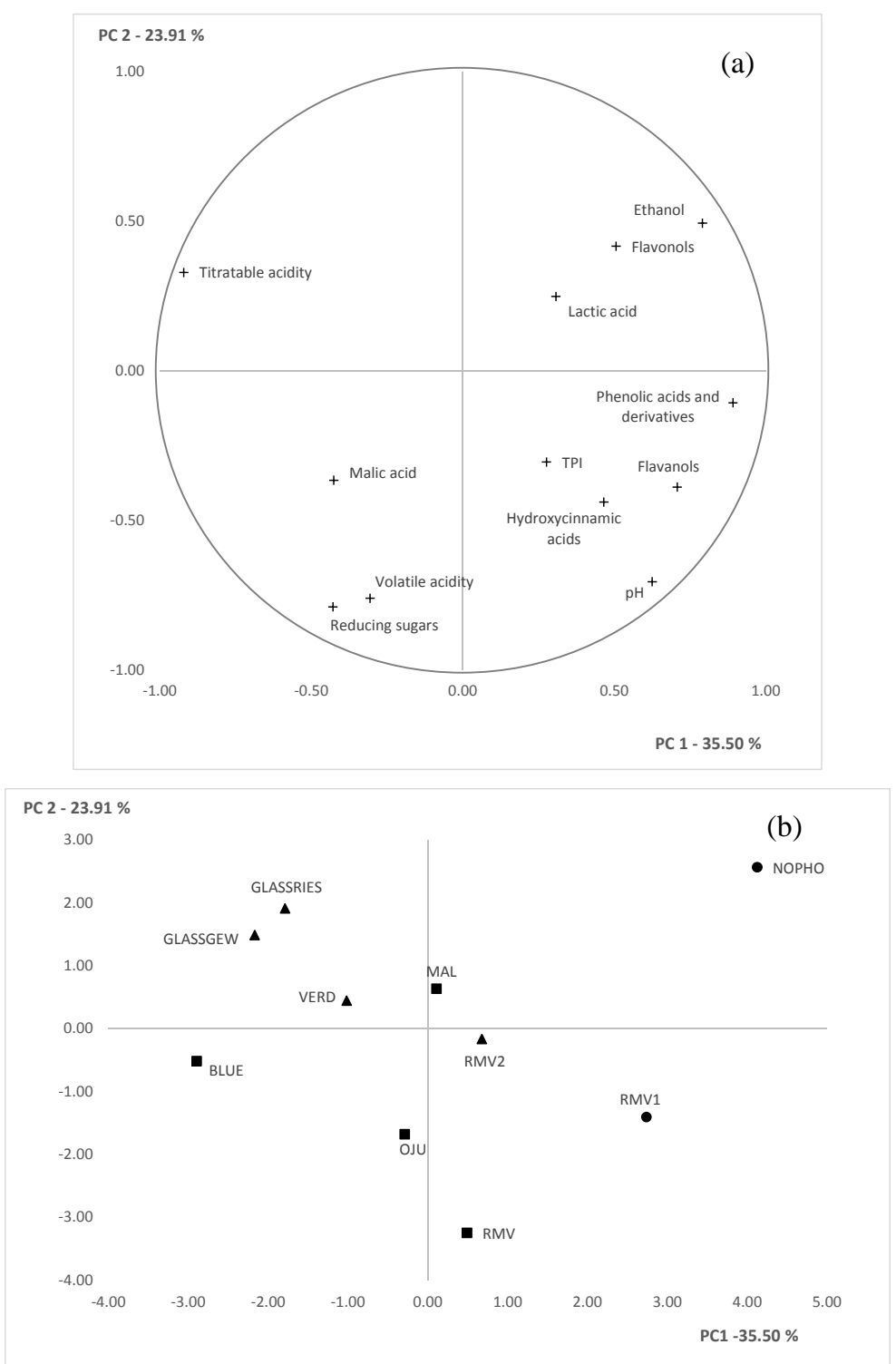

Figure 1. (a) Correlation circle of the PCA for chemical data. (b) Projection of wines on the first two PCs of the PCA for chemical data.

(a) Círculo de correlação da PCA para dados químicos (b) Projeção dos vinhos nas duas primeiras PC da PCA para dados químicos.

In performed hierarchical cluster analysis (HCA), 9 components of the PCA were considered, accumulating $100 \%$ of the original variance. Table VI shows the yielded clusters and the parameters that best characterised each cluster. Figure 1 also shows the different groups. For each group, the wine closest to the centre of gravity was identified as the most typical example of the cluster. 
TABLEVI

Clusters yielded by HCA for chemical data

Clustersobtidospor HCAparadados químicos

\begin{tabular}{clcc}
\hline Cluster & Wines & Positive parameters & Negative parameters \\
\hline $1 \bullet$ & RMV1 $^{*}$, NOPHO $^{*}$ & $\begin{array}{c}\text { Phenolic acids and derivatives, } \\
\text { Hydroxycinnamic acids and derivatives, } \\
\text { flavonols, ethanol }\end{array}$ & Titratable acidity, sugars \\
$2 \boldsymbol{0}$ & OJU $^{*}$, MAL, RMV, BLUE & Volatile acidity, sugars & Flavonols \\
$3 \boldsymbol{\Delta}$ & VERD $^{*}$, GLASSRIES, & - & $\begin{array}{c}\text { pH, Phenolic acids and } \\
\text { derivatives, lactic acid }\end{array}$ \\
\hline & GLASSGEW, RMV2 &
\end{tabular}

The concentrations of different phenolic compounds determined in wine samples were within usual ranges found in white and rosé wines, in which there has not been a maceration with the skins as in the case of target wines. (Pereira et al., 2010; Gonçalves et al., 2012). Cluster 1 was characterised by a greater amount of hydroxybenzoic acids and derivatives, hydroxycinnamic acids and flavonols. It consisted of two wines in which the grape has been harvested in an advanced ripening stage, a late-harvest wine (NOPHO) and a wine from grapes frozen by frost in February (RMV1), which resulted in dehydration of the grapes on-vine. The phenolic composition of wines is affected by the ripening stage of the grapes (Ribéreau Gayon et al., 2006). Some studies have shown that there is an accumulation of polyphenolic compounds until overripening or late harvest in some grape varieties (Ivanova et al., 2011), and a concentration of those compounds is also produced by dehydration on-vine (become raisins) and accumulation in seeds (Rolle et al., 2009). Other authors (Tian et al., 2009a) observed that the contents of hydroxybenzoic acids, hydroxycinnamic acids and flavan-3-ols increased as grape harvest time was delayed to produce icewines in Vidal variety. This cluster is also characterised by a high alcohol content and a lower amount of sugars and titratable acidity, showing a higher fermentation yield with more mature grapes (decrease in acidity).

Cluster 2 was characterised by higher values of volatile acidity and sugars. High volatile acidity was typical in sweet wines production, since yeasts produce higher amounts of acetic acid as a result of the process of maintaining redox balance in response to the osmotic stress imposed by high sugar levels (Nurgel et al., 2004). In any case, the concentrations found in studied sweet wines were below the sensory threshold for acetic acid in icewines, $3.185 \mathrm{~g} / \mathrm{L}$ (Cliff and Pickering, 2006) and even below that th threshold established for table wines, $1.3 \mathrm{~g} / \mathrm{L}$ (Etievant, 1991). This cluster included two icewines (MAL and
BLUE), a supurao wine (dehydration off-vine, OJU) and a wine obtained from grapes frozen in cold chamber (RMV). The first two wines did not have a complete fermentation while the last two wines have experienced a higher concentration of sugars reaching a similar alcoholic degree. This cluster presented intermediate values of phenolic compounds between the other clusters. The icewines of this cluster did not accumulated by dehydration on-vine as many phenolic compounds as icewines of cluster 1, which may be due to environmental, genotypic (variety) or technological (grape pressing) factors. Some studies have observed an increase in the concentration of phenolic compounds in Tempranillo and Merlot grapes (such as hydroxybenzoic acids, esters of hydroxycinnamic acids, flavanols and flavonols) by dehydration or drying inside a chamber under controlled conditions of temperature and humidity (Marquez et al., 2012), which may be similar to the drying process of grapes in supurao winemaking.

Cluster 3 comprised of four artificial icewines made from grapes frozen in freezing chamber. It is characterised by a high acidity and low $\mathrm{pH}$, due to an earlier ripening stage in harvest time, without degradation of acids by overripening. In addition, three of the wines of this group (GLASSRIES, GLASSGEW and VERD) were made from white varieties, and therefore, were intended to achieve higher acidity values. Wines belonging to this cluster presented lower values of hydroxybenzoic acids, so the extraction and concentration of these compounds by freezing have been lower than in the cases of wines of the other two clusters. This fact may also be due to other factors such as variety (white versus red) and grape pressing (diffusion of phenolic compounds from the skin and seeds to the pulp).The presence and concentration of phenolic acids, flavan-3-ols and flavonols could differentiate between methods of artificial and natural icewines, as other authors revealed in Vidal variety (Tian et al., 2009b). 
The low values of lactic acid indicated that wines of this group have not carried out malolactic fermentation, whereas wines belonging to the other two clusters presented quantities of lactic acid that indicated that they have undergone part of malolactic fermentation.

The chemical composition analysed in studied sweet wines had not been significantly different according to the different types of dehydration undergone by grapes (on vine vs. off vine, drying or freezing). So that, more research is needed about the mechanisms and factors that influence the chemical composition during dehydration, apart from the influence of the variety, origin and technology.

\section{Sensory Characterisation}

\section{In-mouth attributes}

According to two way ANOVA with judges as a block factor. The judge effect was significant on all attributes $(p<0.001)$. This effect is commonly found in sensory analysis and can be explained by interindividual differences (Sáenz-Navajas et al., 2011). The wine effect was significant for bitterness $(\mathrm{F}=13.47, \mathrm{p}<0.001)$, acidity $(\mathrm{F}=7.413, \mathrm{p}<0.001)$, sweetness $(\mathrm{F}=13.70, \mathrm{p}<0.001)$, and persistence $(\mathrm{F}=$ $5.709, p=0.004)$, while there were no differences in astringency $(\mathrm{F}=3.308, \mathrm{p}=0.103)$ and global intensity $(\mathrm{F}=1.406, \mathrm{p}=0.186)$. This indicates that these attributes are not useful to characterise and discriminate within this group of wines. Therefore, they were not taken into account for PCA analysis.
Figure 2 shows the two-dimensional PCA plane showing the distribution of the samples on the first two dimensions, as well as the projection of variables. The first two components accumulated more than $90 \%$ of the total variance.

The first PC was mainly correlated with sweetness $(87 \%)$ in positive way and bitterness $(89 \%)$ in negative way, while the second component correlated positively with acidity $(75 \%)$. The wines located to the right of the plane are the sweetest and least bitter, while those located at the top are the most acidic. Pearson's correlation analysis showed that sweetness was inversely correlated with acidity $(\mathrm{F}=-0.698, \mathrm{p}=$ $0.025)$ and bitterness $(\mathrm{F}=-0.667, \mathrm{p}=0.035)$, and persistence was positevely correlated with bitterness $(F=0.745 ; p=0.013)$. This fact agrees with that found in literature, which establishes a suppression of bitterness and acidity by sugar (sweetness) (Jackson, 2009a)

Table VII and Figure 2 show the different clusters found by the HCA, together with the descriptors or attributes that best characterise the groups $(\mathrm{p}<0.05)$.

The first cluster formed by a late harvest wine (NOPHO) stood out for its bitterness, which is related to higher concentrations of hydroxybenzoic acids, hydroxycinnamic acids and flavanols, as previosuly mentioned. In addition, the higher alcohol content in this wine could cause an increase in perceived bitterness (Fischer and Noble, 1994). The wine was located opposite to sweetness, which agrees with less sugar content.

TABLE VII

Clusters yielded by the HCA for in-mouth attributes.

Clustersobtidos por HCA para atributos de boca

\begin{tabular}{|c|c|c|c|}
\hline Cluster & Wines & Positive attributes & Negative attributes \\
\hline $1 \bullet$ & NOPHO $^{*}$ & Bitterness & - \\
\hline 2 घ & OJU*, RMV & - & Acidity \\
\hline $3 \boldsymbol{\Delta}$ & $\mathrm{RMV}^{*}{ }^{*}, \mathrm{RMV} 2, \mathrm{MAL}$ & Persistence & - \\
\hline $4 \bullet$ & $\begin{array}{l}\text { VERD*, GLASSRIES, BLUE, } \\
\text { GLASSGEW }\end{array}$ & Acidity & Persistence \\
\hline
\end{tabular}




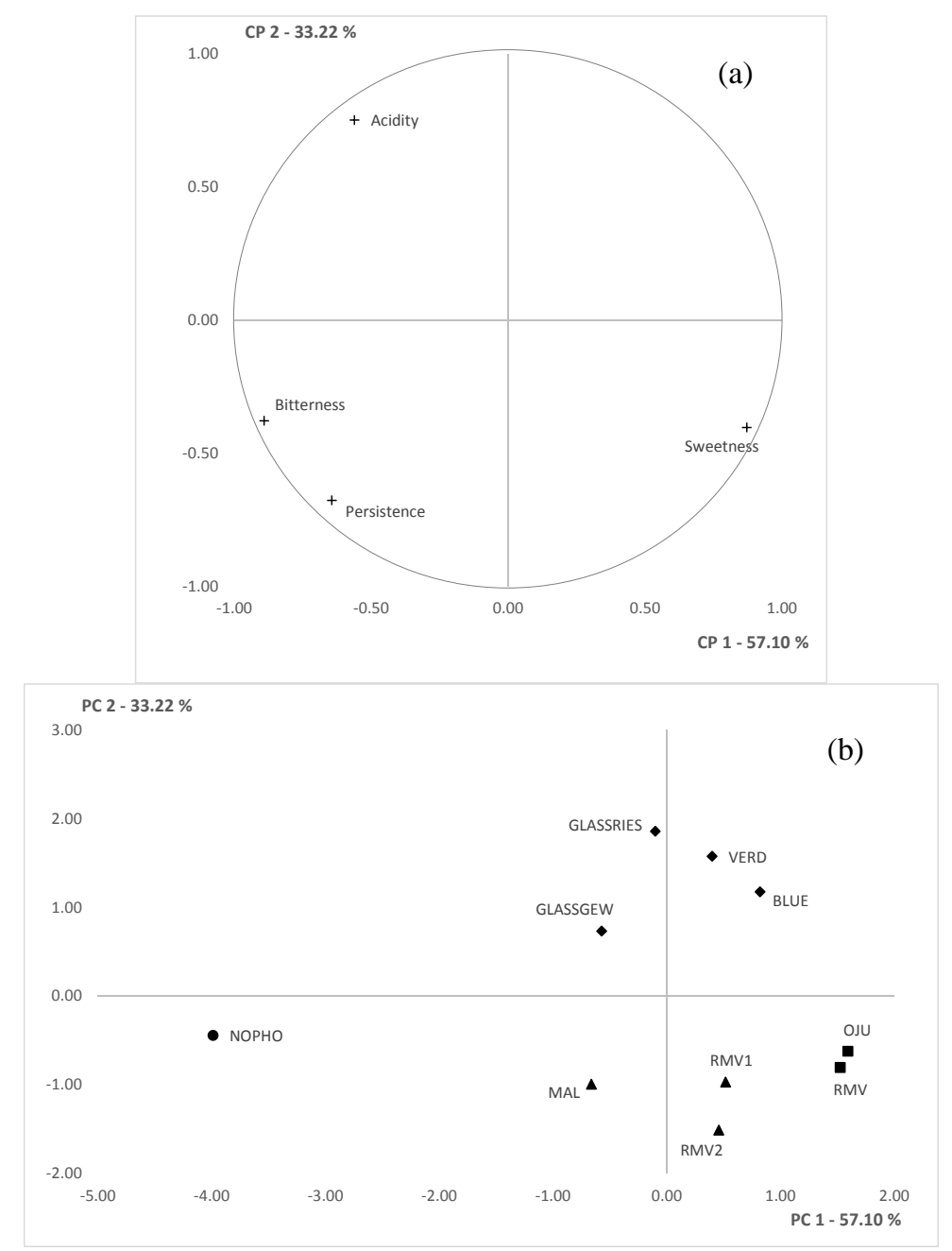

Figure 2. (a) Correlation circle of the PCA for in-mouth attributes. (b) Projection of wines on the first two PCs of the PCA for in-mouth attributes.

(a) Círculo de correlação da PCA para atributos de boca (b) Projeção dos vinhos nas duas primeiras PC da PCA para atributos de boca.

Cluster 2 was characterised by a low perceived acidity $(\mathrm{p}<0.05)$ and sweetness $(\mathrm{p}=0.112)$, located in the lower right part of the PCA plane. It was constituted by supurao wine (OJU) and an artificial ice wine (RMV), which presented a high sugar concentration which could also mask the perceived sensation of acidity in mouth (Jackson, 2009a).

The third cluster formed by natural icewines (RMV1 and MAL) and an artificial icewine (RMV2) was determined by persistence $(\mathrm{p}<0.05)$ and sweetness significant at $90 \%(\mathrm{p}=0.092)$. Those wines presented appreciable amounts of sugar and phenolic compounds (hydroxybenzoic and hydroxycinnamic acids and flavanols), which contribute to sweet and persistent sensations, respectively (Jackson, 2009a; Sáenz-Navajas et al., 2010b). A higher glycerol production takes place in fermentations with highe sugar levels due to osmotic stress that yeasts undergo. Glycerol may contribute to volume in the mouth, in addition to sweetness (Lubbers et al., 2001).

The last cluster consisted of three artificial icewines (GLASSGEW, GLASSRIES and VERD) and one natural icewine (BLUE), all made with white grape varieties. Therefore, this group was highlighted by their perceived acidity in mouth and their low persistence, which might be due to the lower $\mathrm{pH}$ and lower concentration values of phenolic compounds that these white wines presented. The greater acidity could contribute to taste quality by providing a fresh and lively character required in this type of wines,and balancing sweetness. 


\section{Aromatic attributes}

According to two way ANOVA, the effect of wine was significant at the $1 \%$ level $(\mathrm{F}=8.492 ; \mathrm{p}<0.001)$ for the attribute aromatic intensity. Wines with the highest intensity were MAL, RMV2 and NOPHO, which were made with red grapes or mixture of red and white grapes.

Discriminant terms can explain sensory differences found among samples. These terms were identified from the $\chi^{2}$ test performed on frequencies data. 13 terms with a probability of $95 \%$ were selected: citrus fruits, lemon, cherry, dry fruits, prune, dry fig,
banana/English candy, muscat, menthol/fresh, woody, alcohol, sulfur and glue.

In the correspondence analysis (CA) performed on citation frequencies of discriminant terms, the first eight factors were retained and they explained total variance. The projection of the samples and the aromatic terms on the two-dimensional CA plot is shown in Figure 3. The first factor, explained 32\% of the original variance and was mainly defined by the attributes of banana/English candy (82\%) and cherry (67\%), which were opposed to dry fruits (48\%), prune and dry fig (35\%). The second dimension, which accounts for almost $19 \%$ of the original variability, opposed lemon (57\%) and menthol/fresh (23\%) attributes to sulfur and glue (37\%) attributes.

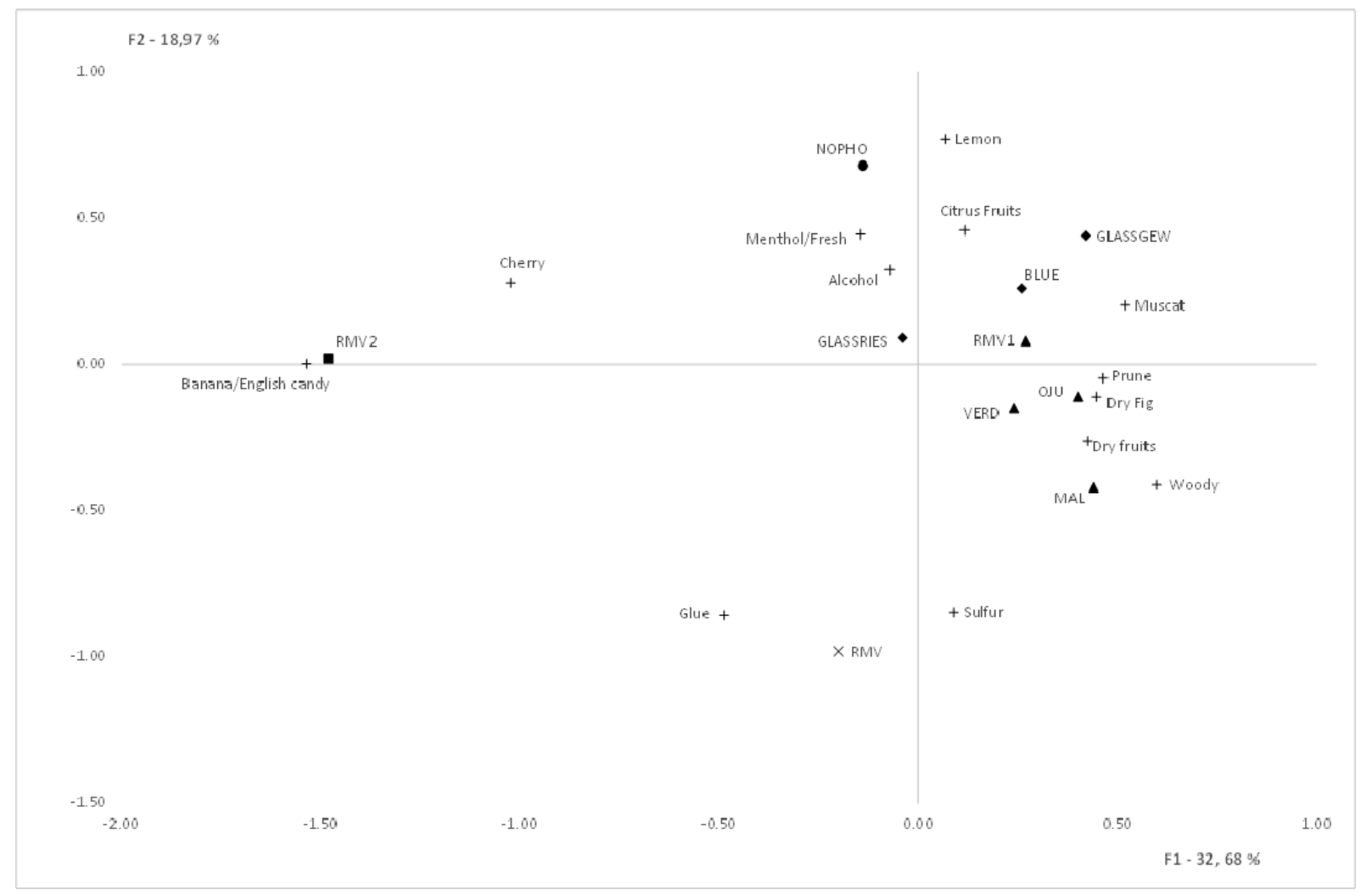

Figure 3. Projection of aroma descriptors and wines in the correspondence analysis space (dimensions 1 and 2).

Projeção de descritores de aroma e vinhos no espaço de análise de correspondência (dimensões 1 e 2).

Hierarchical Cluster Analysis (HCA) revealed that the five-partition option was the most appropriate. Table VIII shows aromatic terms that best characterise samples in each cluster. Figure 3 also shows the different clusters. Cluster 1 was formed by NOPHO, a late harvest wine made from Tempranillo, Viura and Cagazal varieties. It was characterised by alcoholic notes, which agrees with its alcoholic level, and balsamic notes of menthol/fresh and lemon, which makes it a unique wine, as has been also seen in taste and in-mouth description. These aroma nuances could come from a greater presence of terpene varietal aromatic compounds (Setkova et al., 2007) that have been extracted into wine. 
TABLEVIII

Clustersyielded by the HCA for aromaattributes

Clusters obtidos por HCA para atributos do aroma

\begin{tabular}{|c|c|c|}
\hline Cluster & Wines & Descriptores \\
\hline $1 \bullet$ & $\mathrm{NOPHO}^{*}$ & Alcohol, Menthol/fresh, Lemon \\
\hline 2 叫 & $\mathrm{RMV}^{*}$ & Banana/English Candy, Cherry \\
\hline $3 \boldsymbol{\Delta}$ & OJU* ${ }^{*}$ RMV1, MAL, VERD & $\begin{array}{l}\text { Woody, Prune, Dry Fig, Dry } \\
\text { fruits }\end{array}$ \\
\hline $4 \bullet$ & GLASSGEW*, BLUE, GLASSRIES & Citrus fruits, Muscat, Lemon \\
\hline $5 x$ & $\mathrm{RMV}^{*}$ & Glue, Sulfur \\
\hline
\end{tabular}

Cluster 2 also consisted of a singular wine (RMV2, made with frozen grapes in a cold chamber) which stands out for banana/English candy and cherry notes. These descriptors are characteristic of esters (isoamyl acetate presents banana odour), alcohols and aldehydes that are formed in fermentation by yeast activity or by chemical esterification (López de Lerma et al., 2012). It is possible that the precursors of these compounds remained at high levels in Tempranillo red grapes as they were harvested at their optimum ripening stage and then they were concentrated by freezing and pressing. Technological factors such as yeast strain and fermentation conditions could also influenced the resulting aroma.

Cluster 3 consisted of natural ice wines (RMV1 and MAL), supurao wine and an artificial icewine (VERD). Their descriptors agreed with those found in the literature for sweet dessert wines from the Mediterranean area (dry fruits, nuts, raisins) (Genovese et al., 2007; Budic-Leto et al., 2010). Other authors (Reboredo-Rodríguez et al., 2015) have revealed changes in the aromatic profile and volatile composition of grapes for sweet wines production by bifferent dehydration techniques (on vine, off vine in controlled chambers, under the sun, standard conditions in closed environment, freezing), with different varieties and origins, and also have indicated common notes of raisins, figs, caramel, nuts, dry fruits, together with varietals nuances. However, more scientific research on the mechanisms of changes in aromatic profile change is still necessary, due to the enzymatic metabolism of grapes during the dehydration process. Also yeast metabolism during fermentation should be taken into account for the appearance of compounds responsible for these aromas. Other authors have suggested that grapes harvested before and the dried under controlled conditions undergo changes in flavour consistent with those occurring during fruit overripening (Moreno et al., 2008). Although VERD is a white wine made from frozen grapes in cold chamber, without a possible overripening, it presented these typical aromas of dry fuits and raisins, a fact that has also been observed by other authors in Riesling variety when compared to natural ice wines (Clary et al., 2006).

In the fourth cluster, ice wines from white grapes, both natural (BLUE) and artificial (GLASSGEW and GLASSRIES) were grouped, and typical aromas of Riesling and Gewürztraminer varieties predominated (Jackson, 2009b). Overripening notes did not emphasize in its aromatic profile.

The last cluster was formed by RMV (artificial icewine) and it was characterised by sulfur and glue off odours. The increased amounts of sulfur dioxide added to inhibit bacteria due to high sugar concentration in wine have caused the predominance of sulfur notes. As regards glue descriptor, it has been indicated above that a high production of volatile acidity (ethyl acetate, which presents glue aroma) by easts is due to osmotic stress in a medium with large amounts of sugars. Although this wine had the highest value of volatile acidity $(1.19 \mathrm{~g} / \mathrm{L})$ and was below sensory threshold of acetic acid in icewines $(3.1 \mathrm{~g} / \mathrm{L})$, it is possible that the quantity of ethyl acetate present in wine (not quantitatively determined) was above sensory threshold in ice and table wines (0.198 and $0.160 \mathrm{~g} / \mathrm{L}$, respectively) (Cliff and Pickering, 2006).

Through orthonasal aroma description, white wines and late harvest wine have been classified, but no differences have been observed between the other sweet wines from different dehydration processes (supurao, natural or artificial icewines). This finding agrees with a preliminary study, where white wines and late harvest wine were also separated from the 
other wines by a sorting method of sensory analysis (Rosáenz-Oroz et al., 2012).

\section{CONCLUSIONS}

Chemical analyses of conventional oenological parameters using FTIR technique and low molecular weight phenolic compounds using the UPLC-MS technique were suitable in order to characterise sweet natural wines presented in this study. Also, descriptive sensory analysis of in-mouth attributes and aromatic descriptors by citation frequency were adequate for organoleptic characterisation of target natural sweet wines. In view of these results, no significant differences have been established in chemical composition among different dehydration techniques of grapes from which sweet wines were obtained. Nevertheles, most of artificial ice wines (off-vine dried grapes) presented lower concentrations of phenolics, so they could be differentiated from wines obtained from on-vine dried grapes. Through sensory analysis of in-mouth attributes and evaluated aromatic descriptors, no significant differences among different processes of grapes dehydration have been observed. Aromatic

\section{REFERENCES}

AOAC, 2016. Official Methods of Analysis $20^{\text {th }}$ Edition. 2200 p. Association of Analytical Communities, Gaithersburg.

Bellincontro A., De Santis D., Botondi R., Villa I., Mencarelli F., 2004. Different postharvest dehydration rates affect quality characteristics and volatile compounds of Malvasia, Trebbiano and Sangiovese grapes for wine production. J. Sci. Food Agric., 84, 1791-1800.

Bowen A., Reynolds A., Lesschaeve I., 2016. Harvest date and crop level influence sensory and chemical profiles of Ontario Vidal blanc and Riesling icewines. Food Res. Int., 89, 591-603.

Budic-Leto I., Zdunic G., Banovic M., Kovacevic-Ganic K., Tomic-Potrebujes I., Lovric T., 2010. Fermentative aroma compounds and sensory descriptors of traditional croatian dessert wine Prosek from Plavac mali cv. Food Technol. Biotechnol., 48, 530-537.

Campo E., Do B., Ferreira V., Valentin D., 2008. Aroma properties of young Spanish monovarietal white wines: a study using sorting task, list of terms and frequency of citation. Aust. J. Grape Wine Res., 14, 104-115.

Clary C., Gamache A., Cliff M., Fellman J., Edwards C., 2006. Flavor and aroma attributes of riesling wines produced by freeze concentration and microwave vacuum dehydration. J. Food Process. Preserv., 30, 393-406.

Cliff M.A., Pickering G.J., 2006. Determination of odour detection thresholds for acetic acid and ethyl acetate in ice wine. J. Wine Res., 17, 45-52.

Crandles M., Reynolds A., Khairallah, R. B., 2015. The effect of yeast strain on odor active compounds in Riesling and Vidal blanc icewines. LWT - Food Sci. Technol., 64, 243-258. descriptors of dry fruits and prune were observed in most wines from on-vine and off-vine dehydration. However, sensory analysis was able to differentiate late harvest wine, and white wines, without discrimination of dehydration techniques in this last group. More studies will be needed on factors affecting sensory parameters during dehydration.

\section{ACKNOWLEDGMENTS}

The authors want to thank Instituto de Estudios Riojanos (IER) for support; María-Pilar Sáenz Navajas and Purificación Fernández-Zurbano (from Laboratory for Aroma Analysis and Oenology, University of La Rioja and Institute of Vine and Wine Sciences) for their collaboration and assistance; University of La Rioja and its Laboratory Service for the infrastructure provided; Gonzalo Ayala (Bodegas Ramón de Ayala Lete e Hijos); Daniel López and Faith B. Matthews for English revision; Izaskun Garrido, María Íñiguez, Franz Zirena, and Vesna Terrero for their help and collaboration in carrying out the work.

D'Onofrio C., 2013. Changes in volatile vompounds. In: Sweet, Reinforced and Fortified Wines. 91-104. Mencarelli F., Tonutti P. (eds.), John Wiley \& Sons Ltd.,West Sussex.

Etievant P., 1991. Wine. In: Volatile Compounds in Food and Beverages. 145-149. Maarse H (ed.), Marcel Dekker, New York.

Fang F., Li J., Zhan P., Tang K., Wang W., Pan Q., Huang W., 2008. Effect of grape variety, harvest date, fermentation vessel and wine ageing on flavonoid concentration in red wines. Food Res. Int., 41, 53-60.

Figueiredo-González M., Cancho-Grande B., Simal-Gándara J., 2013. Effects on colour and phenolic composition of sugar concentration processes in dried on- or dried off-vine grapes and their aged or not natural sweet wines. Trends Food Sci. Technol., 31, 36-54.

Fischer U., Noble A., 1994. The effect of ethanol, catechin concentration and $\mathrm{pH}$ on sourness and bitterness of wine. Am. $J$. Enol. Vitic., 45, 6-10.

Genovese A., Gambuti A., Piombino P., Moio L., 2007. Sensory properties and aroma compounds of sweet Fiano wine. Food Chem., 103, 1228-1236.

Gonçalves J., Silva C., Castilho P., Câmara J. , 2012. An attractive,sensitive and high-throughput strategy based on microextraction by packed sorbent followed by UHPLC-PDA analysis for quantification of hydroxybenzoic and hydroxycinnamic acids in wines. Microchem. J., 106, 129-138.

González-Álvarez M., Noguerol-Pato R., González-Barreiro C., Cancho-Grande B., Simal-Gándara J., 2013. Sensory quality control of young vs. aged sweet wines obtained by the techniques of both postharvest natural grape dehydration and fortification with spirits during vinification. Food Anal. Methods, 6, 289-300. 
Gonzalez-Hernandez M., Avizcuri-Inac J., Dizy M., FernándezZurbano P., 2014. Ultra performance liquid chromatography coupled to Ultraviolet-Vis and mass spectrometry detector for screening of organic acids and polyphenols in red wine. In: HighPerformance Liquid Chromatography (HPLC): Principles, Practices and Procedures. 267-297. Yuegang Zuo (ed.), Nova Science Publishers, Dartmouth.

Hermann A., 2014. Icewine Authentication by $\delta 180$ Measurements. Am. J. Enol. Vitic., 65, 499-503.

ISO, 1977. ISO NORM 3591:1977 Sensory analysis: apparatus wine tasting glass. 24 p. International Organization for Standardization, Geneva.

Ivanova V., Stefova M., Vojnoski B., Dörnyei A., Márk L., Dimovska V., 2011. Identification of polyphenolic compounds in red and white grape varieties grown in R. Macedonia and changes of their content during ripening. Food Res. Int., 44, 2851-2860.

Jackson R., 2009a. Taste and mouth-feel sensations. In: Wine Tasting - A Professional Handbook. 129-175. R. Jackson (ed.), Academic Press, San Diego.

Jackson R., 2009b. Styles and types of wine. In: Wine Tasting - A Professional Handbook. 349-386. R. Jackson (ed.), Academic Press, San Diego.

Kilmartin P. A., Reynolds A., Pagay V., Nurgel C., Johnson, R., 2007. Polyphenol content and browning of Canadian icewines. $J$. Food Agric. Environ., 5, 52-57.

Lan Y.B., Quian X., Yang Z.J., Xiang X.F., Yang W.X. Liu T., Zhu B.Q.; Pan Q.H., Duan C.Q., 2016. Striking changes in volatile profiles at sub-zero temperatures during over-ripening of 'Beibinghong' grapes in Northeastern China. Food Chem., 212, $172-182$.

Li J.C., Li S.Y., He F., Yuan Z.Y., Liu T., Reeves M.J., Duan, C.Q., 2016. Phenolic and chromatic properties of Beibinghong red ice wine during and after vinification. Molecules, 21, 431-447.

López de Lerma N., García-Martínez T., Moreno J., Mauricio J., Peinado R., 2012. Volatile composition of partially fermented wines elaborated from sun dried Pedro Ximénez grapes. Food Chem., 135, 2445-2452.

Lubbers S., Verret C., Voilley A., 2001. The efect of glycerol on the perceived aroma of a model wine and a white wine. LWT Food Sci. Technol., 34, 262-265.

Luo L., Cui Y., Zhang S., Li L., Suo H., Sun B., 2017. Detailed phenolic composition of Vidal grape pomace by ultrahighperformance chromatography-tandem mass spectrometry. $J$. Chromatogr. B, 1068-1069, 201-209.

Marquez A., Serratosa M., Lopez-Toledano A., Merida J., 2012. Colour and phenolic compounds in sweet red wines from Merlot and Tempranillo grapes chamber-dried under controlled conditions. Food Chem., 130, 111-120.

Moreno J., Cerpa-Calderón F., Cohen S., Fang Y., Qian M., Kennedy J., 2008. Effect of postharvest dehydration on the composition of pinot noir grapes (Vitis vinifera L.) and wine. Food Chem., 109, 755-762.

Morineau A., Lebart L., Piron M., 1995. Statistique exploratoire multidimensionnelle. 439 p. Dunod, Paris.

Nurgel C., Pickering G., Inglis D., 2004. Sensory and chemical characteristics of Canadian ice wines. J. Sci. Food Agric., 84, 16751684

OIV, 2016. Recueil des methodes internationales d'analyses des vines et des moûts. 534 p. OIV, París.
OIV, 2018. OIV Code sheet - Issue 2018/01 I.1.4-7. International Code of Oenological Practices. 432 p. OIV, París

Pereira V., Câmara J., Cacho J., Marques J., 2010. HPLC-DAD methodology for the quantification of organic acids, furans and polyphenols by direct injection of wine samples. J. Sep. Sci., 33, 1204-1215.

Ramos R., Andrade P.B., Seabra R., Pereira C.F., 1999. A preliminary study of non-coloured phenolics in wines of varietal white grapes (códega, gouveio and malvasia fina): Effects of grape variety, grape maturation and technology of winemaking. Food Chem., 67, 39-44.

Reboredo-Rodríguez P., González-Barreiro C., Rial-Otero R., Cancho-Grande B., Simal-Gándara J., 2015. Effects of sugar soncentration processes in grapes and wine aging on aroma compounds of sweet wines-A review. Crit. Rev. Food. Sci. Nutr. 55, 1053-1073.

Ribéreau Gayon P., Glories Y., Maujean A., Dubourdieu D., 2006. Phenolic compounds. In: Handbook of Enology Volume 2: The Chemistry of Wine: Stabilization and Treatments. 141-203. John Wiley \& Sons Ltd., West Sussex.

Rolle L., Torchio F., Giacosa S., Gerbi V.,2009. Modifications of mechanical characteristics and phenolic composition in berry skins and seeds of Mondeuse winegrapes throughout the on-vine drying process. J. Sci. Food Agric., 89, 1973-1980.

Rosáenz-Oroz D., Martínez-Ruiz R., Vaquero-Fernández L., 2012. Elaboración de vinos de hielo en La Rioja: impacto de la congelación natural y artificial. Zubía, 30, 199-223.

Sáenz-Navajas M.P., Campo E., Fernández-Zurbano P., Valentin D., Ferreira V., 2010b. An assessment of the effects of wine volatiles on the perception of taste and astringency in wine. Food Chem., 121, 1139-1149.

Sáenz-Navajas M.P., Ferreira V., Dizy M., Fernández-Zurbano P., 2010a. Characterization of taste-active fractions in red wine combining HPLC fractionation, sensory analysis and ultra performance liquid chromatography coupled with mass spectrometry detection. Anal. Chim. Acta, 673, 151-159.

Sáenz-Navajas M.P., Martín-López C., Ferreira V., FernándezZurbano P., 2011. Sensory properties of premium Spanish red wines and their implication in wine quality perce. Aust. J. Grape Wine Res., 17, 9-19.

Schwarz M., Rodríguez M., Guillén D., Barroso C., 2009. Development and validation of UPLC for the determination of phenolic compounds and furanic derivatives in Brandy de Jerez. $J$. Sep. Sci., 32, 1782-1790.

Scienza A., 2013. Sweet wines: The essence of european civilization. In: Sweet, Reinforced and Fortified Wines, 5-25. Mencarelli F., Tonutti P. (eds.), Wiley-Blackwell, West Sussex.

Serratosa M.P., Marquez A., Lopez-Toledano A., Medina M., Merida J., 2011. Changes in hydrophilic and lipophilic antioxidant activity in relation to their phenolic composition during the chamber drying of red grapes at a controlled temperature. J. Agric. Food Chem., 59, 1882-1892.

Setkova L., Risticevic S., Pawliszyn J., 2007. Rapid headspace solid-phase microextraction-gas chromatographic-time-of-flight mass spectrometric method for qualitative profiling of ice wine volatile fraction II: Classification of Canadian and Czech ice wines using statistical evaluation of the data. J. Chromatogr. A, 1147, $224-240$

Synos K., Reynolds A.G., Bowen, A., 2015. Effect of yeast strain on aroma compounds in Cabernet franc icewines. LWT - Food Sci. Technol., 64, 227-235. 
Tang K., Li J., Wang B., Ma L., Xu Y., 2013. Evaluation of nonvolatile flavor compounds in Vidal icewine from China. Am. J. Enol. Vitic., 64, 110-117.

Tian R.R., Li G., Wan S.B., Pan Q.H., Zhan J.C., Li J.M., Zhang Q.H., Huang W.D., 2009b. Comparative study of 11 phenolic acids and five flavan-3-ols in cv. Vidal: impact of natural icewine making versus concentration technology. Aust. J. Grape Wine Res., 15, 216-222.
Tian R.R., Pan Q.H., Zhan J.C., Li J.M., Wan S.B., Zhang Q.H., Huang W.D., 2009a. Comparison of phenolic acids and flavan-3ols during wine fermentation of grapes with different harvest times. Molecules, 14, 827-838.

Vidal S., Francis L., Noble A., Kwiatkowski M., Cheynier V., Waters E., 2004. Taste and mouth-feel properties of different types of tannin-like polyphenolic compounds and anthocyanins in wine. Anal. Chim. Acta, 513, 57-64. 